\title{
Autologous MAGE-A3/A6-specific TCR Gene-engineered Lymphocytes KITE-718
}

National Cancer Institute

\section{Source}

National Cancer Institute. Autologous MAGE-A3/A6-specific TCR Gene-engineered

Lymphocytes KITE-718. NCI Thesaurus. Code C135534.

Human autologous T-lymphocytes genetically modified to express a T-cell receptor (TCR) that specifically targ ets human melanoma-associated antigen A3 (MAGE-A3) and MAGE-A6 (MAGEA3/A6; MAGE-A3/A6), with potential antineoplastic activity. Peripheral blood mononuclear cells (PBMCs) are isolated from a patient, transduced with a gene expressing a TCR specific for the MAGE-A3/A6 antigens, expanded ex vivo, and reintroduced into the patient. Then, the autologous MAGE-A3/A6-specific TCR gene eng ineered lymphocytes KITE-718 target and bind to tumor cells expressing the MAGEA3 and/or MAGE-A6 antigens. This halts the growth of and kills MAGE-A3/A6-expressing cancer cells. The tumor-associated antigens MAGE-A3 and MAGE-A6 are overexpressed on a variety of tumor cell types. 